\title{
FURLABLE REFLECTOR CONCEPT FOR SMALL SATELLITES
}

\author{
A.G. Tibert $^{*}$ and S. Pellegrino ${ }^{\dagger}$ \\ Department of Engineering, University of Cambridge, \\ Trumpington Street, Cambridge, CB2 1PZ, U.K.
}

\begin{abstract}
$\underline{\text { Abstract }}$
A new concept for deployable mesh reflectors is presented. It consists of a deployable ring structure and two identical cable nets (front and rear nets) connected by tension ties. The reflecting mesh is attached to the front net. The concept has been demonstrated by means of simple demonstrator hardware. A preliminary design of a $3 \mathrm{~m}$ diameter, $10 \mathrm{GHz}$ reflector with a focal length to diameter ratio $F / D=0.4$ that could be packaged within an envelope of $0.1 \times 0.2 \times 0.8 \mathrm{~m}^{3}$ is presented.
\end{abstract}

\section{Introduction}

There is currently a growing interest in low-cost deployable appendages for small satellites, and the development of structures for such applications is a lively area of research in the Deployable Structures Laboratory, at Cambridge University.

The work presented in this paper was motivated by an application requiring a $3 \mathrm{~m}$ diameter parabolic reflector to be launched alongside a $0.6 \times 0.6 \times 0.8 \mathrm{~m}^{3}$ bus, packaged within an envelope of $0.1 \times 0.2 \times 0.8 \mathrm{~m}^{3}$, the last dimension is a hard limit. The reflector is to have a focal length to diameter ratio $F / D=0.4$ and operate at a frequency of $10 \mathrm{GHz}$. Excluding inflatables, which still cannot be regarded as a mature technology, none of the existing deployable reflector systems is suitable for this application. It was considered that an adaptation of the AstroMesh concept (Thomson 1997) offers the greatest potential for meeting the requirements with a low-cost system.

In the AstroMesh, see Figure 1, the reflective mesh is attached to a network of thin cables, or tapes with high axial stiffness that approximates to a paraboloid; the cables are prestressed to form a stiff and accurate structure. The size of the triangles forming the cable network is chosen sufficiently small to achieve the required accuracy. This concept is known as a tension truss and was invented by Miura (1986). The forces required to prestress the ca- ble net are provided by a series of springs, called tension ties in Figure 1, connecting the network to an identical rear net. Both nets are connected around the edge to a deployable ring truss with telescopic diagonals.

Note that the height of the ring truss is given by the depth of the two nets plus their separation. Although the rear net can be made less deep, say half the depth of the front net, by accepting larger forces on the ring, a reflector with small $F / D$ requires a high ring. For example, for a reflector depth $H=0.46 \mathrm{~m}$ and $D=3 \mathrm{~m}$ an AstroMeshtype truss divided into 18 segments would have a packaged height of more than $1.2 \mathrm{~m}$. An alternative ring configuration, based on a pantograph with, again, 18 bays would have a height of $0.9 \mathrm{~m}$. However, this requires a large number of joints.

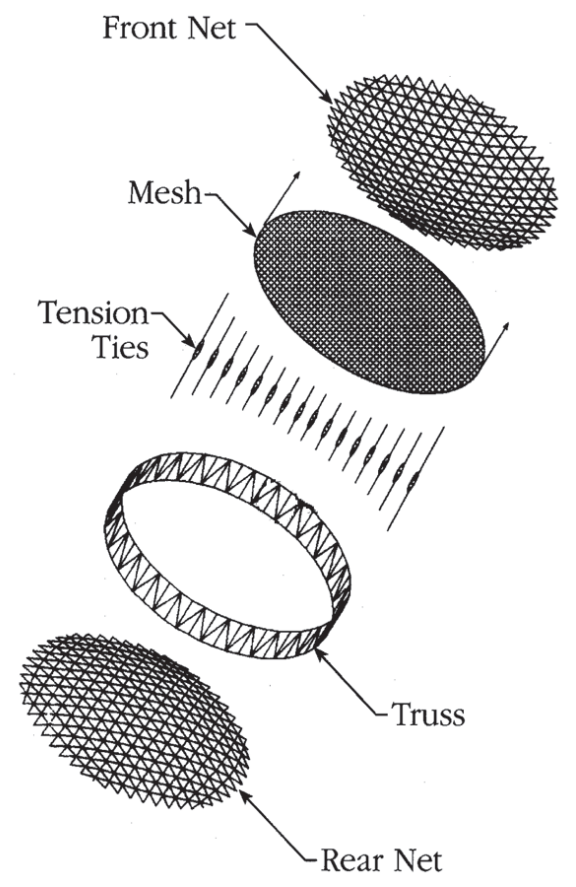

Figure 1: AstroMesh concept (from Thomson 1997).

\footnotetext{
* Junior Visitor. Permanent address: Department of Structural Engineering, Royal Institute of Technology, SE 10044 Stockholm, Sweden

${ }^{\dagger}$ Professor of Structural Engineering, Associate Fellow AIAA.

Copyright (C) 2001 by S. Pellegrino. Published by the American Institute of Aeronautics and Astronautics, Inc. with permission.
} 
For the reasons stated above it was concluded that a new concept was needed in order to meet the present requirements. Hence, this paper presents a new concept that has been developed. Simple demonstrator hardware has been made to illustrate the concept and demonstrate its viability. Finally, a preliminary design of a $3 \mathrm{~m}$ reflector that would meet all of the requirements is presented.


Figure 2: New concept.

\section{New Concept}

The proposed reflector structure is based on the tension truss concept. Like the AstroMesh, it is composed of three main parts:

- a deployable ring structure;

- two identical cable nets (front and rear nets) connected by tension ties;
- the reflecting mesh, attached to the front net.

Although the concept is a general one, for clarity it will be explained with reference to the particular example shown in Figure 2. Basically, we are dealing with a structure consisting of a large number of cable elements and constant-tension springs with only six struts (compression elements).

Figure 2(a) highlights the 18 cable elements and 6 struts that form the deployable ring structure. This is a well-known "tensegrity structure" belonging to a family invented in 1948 by the sculptor Kenneth Snelson and R. Buckminster Fuller. Recently, Duffy et al. (2000) have pointed out the potential usefulness of deployable tensegrity structures for spacecraft applications. Two important features of tensegrity structures are that:

- there is no connection between compression elements;

- the connections between compression and tension members are simple.

These features make them particularly attractive in applications requiring low-weight, low-cost deployables that can be packaged very compactly. A disadvantage of standard tensegrity structures is that they are very flexible, due to the existence of internal mechanisms of inextensional deformation, as will be shown next. However, we have obtained a new solution that avoids this problem.

Consider the pin-jointed structure shown in Figure 3, whose layout is identical to the ring structure in Figure 2. The top six joints lie at the corners of a regular hexagon and the bottom six joints lie at the corners of an identical hexagon. Each joint is connected by bars to the two neighbouring joints in the same hexagon, and also to two joints of the top hexagon. Note that it is not connected to the joint directly above, but to the next and the second next joints, in an anti-clockwise sense.

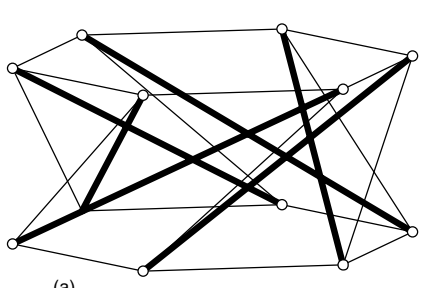

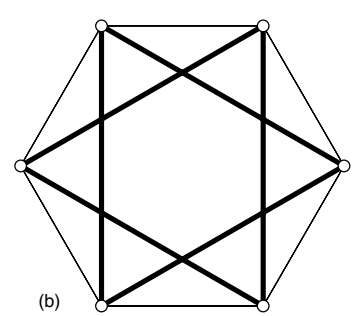

Figure 3: Hexagonal tensegrity module; (a) threedimensional view; (b) top view.

This structure has $j=12$ joints and $b=24$ bars. To investigate its static and kinematic properties we use the extended Maxwell's rule (Calladine 1978)

$$
3 j-b=m-s
$$

where 
- $m=$ number of independent inextensional mechanisms, and

- $s=$ number of independent states of self-stress

Substituting the values of $j$ and $b$ into Equation 1 we obtain

$$
m-s=12
$$

It can be shown that this structure has one state of selfstress, $s=1$, where the six longer bars connecting the two hexagons are in compression and all other members are in tension. Therefore, from Equation 2 we conclude that $m=13$ and, since six mechanisms will be rigid-body motions of the whole structure, this leaves seven internal mechanisms. These mechanisms can be stiffened by prestressing the structure, but this will only provide a relatively small amount of stiffness.

Because this structure can be prestressed, as described, a deployable version can be made quite easily. The state of prestress will require only six members to carry compressive forces, all other members are in tension and therefore-instead of using bars-they can be replaced with cables. Then, if the struts are collapsible, e.g. either telescopic or foldable at a series of hinge points, the whole structure can be folded.

This structure, however, has seven internal mechanisms, which is clearly undesirable. Therefore, we have modified it by connecting two identical triangulated structures to the hexagons, as shown in Figure 2(b); the layout of these nets is more clearly shown in Figure 4.

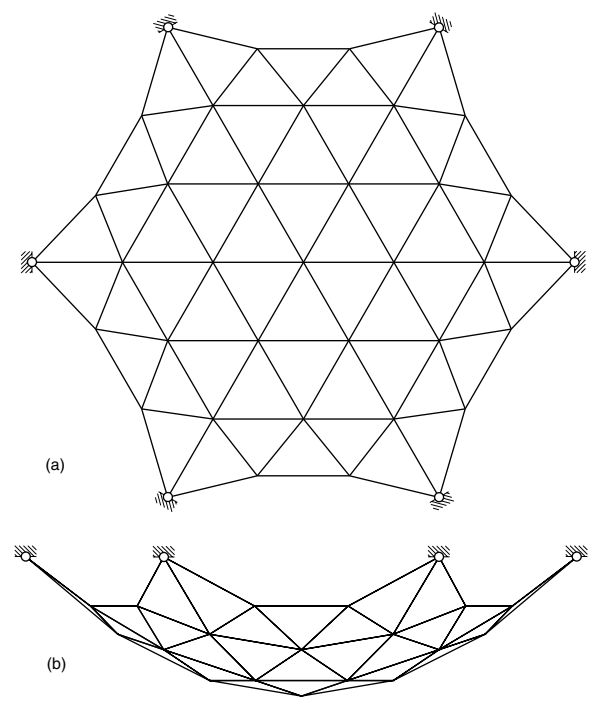

Figure 4: (a) Top and (b) side views of front and rear nets.

The layout of these nets can be defined in many different ways, for example it could be optimised such that all triangles have equal area and are as close as possible to equilateral. The particular layout that was chosen is based on a simple two-dimensional, regular tessellation of equilateral triangles that is obtained by dividing each side of a hexagon into three. Then, the outermost triangles were distorted to form a catenary-like edge for the net to improve the force distribution in it. Finally, all nodes were projected onto a paraboloid, see the Appendix.

Consider the structure consisting of the original ring structure plus the two triangulated nets; its static and kinematic properties are investigated as follows

- Number of joints: there are 6 joints in the symmetry unit of each net, hence

$$
j=2 \times(1+6 \times 6)=74
$$

- Number of bars: there are 15 bars in the symmetry unit of each net, plus the 24 bars of the ring structure, hence

$$
b=2 \times(15 \times 6)+24=204
$$

Substituting Equations 3 and 4 into Equation 1 we obtain

$$
m-s=18
$$

Since the state of self-stress is still statically possible, but no additional states of self-stress have been created, we have $s=1$. Hence, $m=19$ and, of these mechanisms, 6 are rigid-body motion and 13 internal. The 13 internal mechanisms can be removed by adding 13 bars to the structure, as shown in Figure 2(c). The resulting structure has $m=6$, and hence only rigid-body mechanisms, and $s=1$.

To realise this structure in practice we need to find a way of prestressing the two nets. The obvious way of doing it is to connect corresponding nodes of the two nets with a series of tension ties that apply equal forces. It turns out that this is not an ideal solution because

1. large compressive forces are induced in the cables of the ring structure, which need to be counteracted by increasing the level of prestress of the ring; this would further increase the compression in the struts;

2. 12 of the 13 additional members shown in Figure 2(c) are not pre-tensioned;

It was found that all of these issues can be resolved by modifying the configuration of the ring structure. Instead of using the original configuration, where the two hexagons are directly one above the other as shown in Figure 3(b), one hexagon is rotated through a small angle, Figure 5 . 


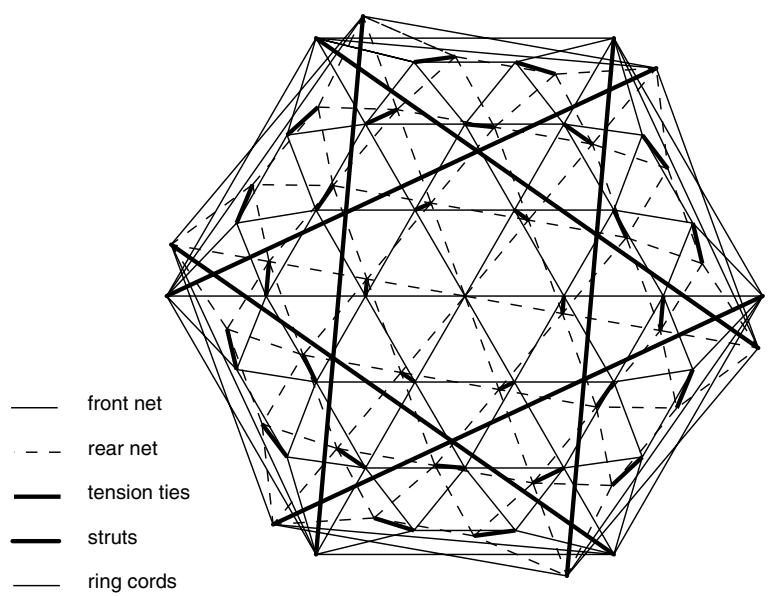

Figure 5: Complete structure, additional 12 members not shown.

By itself, the resulting ring structure can no longer be prestressed, as $s=0$ and hence, from Equation 2, $m=12$. However, when the structure is considered in its entirety, including the prestressing forces applied by the tension ties, the following is found.

- To obtain a structure free of internal mechanisms only 12 additional members are required, not 13 , hence the member connecting the centres of the two nets can be replaced with a tension tie.

- For a $10^{\circ}$ anti-clockwise rotation of the upper hexagon with respect to the bottom hexagon-as shown in Figure 5-all of the cables are in a state of tension.*



Figure 6: Forces in the two nets due to tension tie loads of $1 \mathrm{~N}$ on the inner nodes and $2 \mathrm{~N}$ on the edge nodes.
Figure 6 shows the force distribution in the two nets; the corresponding forces in the ring structure are $-68.8 \mathrm{~N}$ in the struts, $+25.9 \mathrm{~N}$ in the cables forming the hexagons, and $+39.5 \mathrm{~N}$ in the six cables linking the hexagons.

\section{Configuration of Tensegrity Reflector}

In addition to studying the statical and kinematical properties of the reflector structure, it is necessary to analyse the effect of different design parameters on the magnitude and distribution of the forces within the structure. Our aim is to obtain a fairly uniform distribution of forces in the net and to avoid large forces in the supporting structure, particularly the struts. The configuration study is divided into two parts. First, the influence of the sag-to-span ratio of the net edges and the tension ties forces on the forces in the net is investigated. Then, for some particular values of the forces in the tension ties and a particular sag-to-span ratio, the effect of the relative rotation of the hexagons on the forces in the nets and the ring structure is considered.

A detailed description of the procedure used for generating the triangular net mesh and how the sag-to-span ratio is defined are given in the Appendix, at the end.

\section{Sag-to-Span Ratio and Tension Tie Forces}

Throughout this first study the tension ties are represented by vertical loads on the joints of the net, see Figure 7 . We begin by checking the statical and kinematical properties of the three-ring cable net in Figure 7. From the Appendix, the number of joints is

$$
b=6 \frac{3(1+3 \times 3)}{2}=90
$$

and the number of bars is

$$
j=1+6 \frac{3(1+3)}{2}=37
$$

The extended Maxwell's rule, Equation 1, yields

$$
m-s=21
$$

From the synclastic shape of the net, it is obvious that no state of self stress can be sustained giving $s=0$. By fixing 6 joints in space we get $m=3$. Hence, we need to fix another three degrees of freedom to eliminate the internal mechanisms. Following Pellegrino (1993) we have analysed the equilibrium matrix of the structure and thus computed three independent mechanisms. By looking at plots of these mechanisms we decided to fix one edge joint radially and tangentially, i.e. two perpendicular in-plane directions, and its neighbouring edge joint radially. This gives a statically and kinematically determinate structure.

\footnotetext{
* Note that the forces in the outer ties have been set to twice the value of the internal ties.
} 


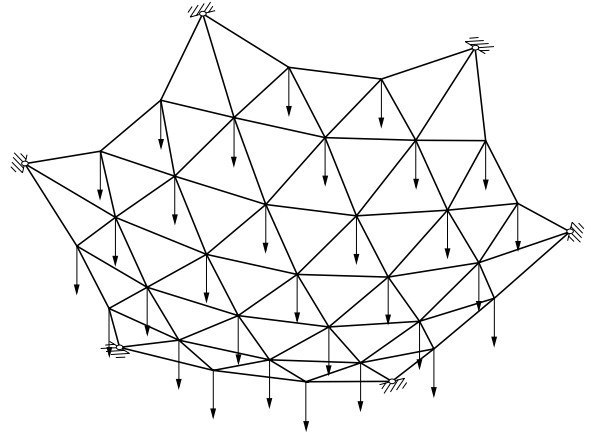

Figure 7: Loads applied to cable net to study the influence of sag-to-span ratios on the force pattern.

The cable net in Figure 7 was analysed for three sag-tospan ratios: 5, 10 and $15 \%$. For each ratio the initial setting of the tension tie forces was $1 \mathrm{~N}$ everywhere, which is most practical as identical constant-tension springs would be used in all of the tension ties. However, if the force pattern in the net is irregular or, worse, some elements are in compression, the tension tie forces have to be adjusted.

The results for a $5 \%$ sag-to-span ratio are shown in Figure 8. For the case where the tension tie forces are all equal to $1 \mathrm{~N}$, Figure 8(a), some members are in compression. By increasing the edge forces the compressive forces gradually become smaller and then tensile, Figure 8(b)-(d), as the edge forces are increased. An almost uniform force distribution is obtained for edge forces of $4 \mathrm{~N}$, however the largest force in the edge cable is now over $15 \mathrm{~N}$.


Figure 8: Forces in a net with 5\% sag-to-span ratio. Loads on inner nodes: $1 \mathrm{~N}$; loads on edge nodes (a) $1 \mathrm{~N}$, (b) 2 $\mathrm{N}$, (c) $3 \mathrm{~N}$, (d) $4 \mathrm{~N}$.

When the sag-to-span ratio is increased to $10 \%$ there is still compression for tension tie forces of $1 \mathrm{~N}$, Figure 9(a). However, as the force in the edge ties is increased to $2 \mathrm{~N}$ an acceptable distribution of net forces is obtained and the edge forces are smaller than for the 5\% sag-tospan ratio, Figure 9(b). The range of the inner net forces is $0.75-2.69 \mathrm{~N}$.
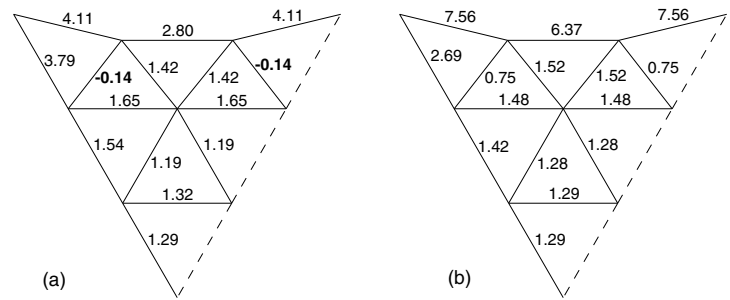

Figure 9: Forces in a net with $10 \%$ sag-to-span ratio. Loads on inner nodes: $1 \mathrm{~N}$; loads on edge nodes (a) $1 \mathrm{~N}$, (b) $2 \mathrm{~N}$.

Increasing the sag-to-span ratio further to $15 \%$ yields no compressed elements even for the case of uniform $1 \mathrm{~N}$ tension tie loads, Figure 10(a). By increasing to $2 \mathrm{~N}$ the forces in the edge ties gives a very uniform force pattern, in the range $1.27-2.08 \mathrm{~N}$, and the edge cable forces are slightly smaller than in the previous case.
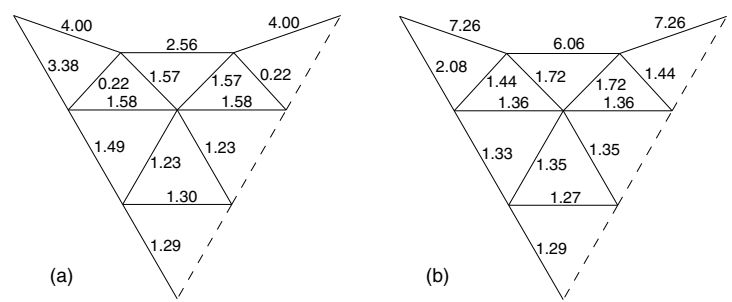

Figure 10: Forces in a net with $15 \%$ sag-to-span ratio. Loads on inner nodes: $1 \mathrm{~N}$; loads on edge nodes (a) $1 \mathrm{~N}$, (b) $2 \mathrm{~N}$.

Although a sag-to-span ratio of $15 \%$ gives a better force pattern than the $10 \%$ ratio, the further loss of reflecting area is not justified, hence $10 \%$ is the value that is selected.

\section{Rotation of Hexagons}

Next, the effect on the prestress distribution of a relative rotation $\theta$ between the hexagons of the ring structure is analysed, assuming that the cable nets have a fixed sagto-span ratio of $10 \%$. The tension tie force is $1 \mathrm{~N}$ on the inner joints and $2 \mathrm{~N}$ on the edge joints, giving the force distribution shown in Figure 9(b) for $\theta=0$. However, when the hexagons are rotated, the force distribution in a bay of the net is no longer symmetric, Figures 11 and 12 .

Net forces

Figure 11 plots the variation in the forces of the inner net 
elements with the rotation of the hexagons. The forces in the radial cables 1, 3 and 8 are approximately constant, for the range of $\theta$ displayed. The other cable forces-except for cables 9 and 10-are within 0.5-1.5 N. Most importantly, cable 9 becomes compressed at $\theta \approx 28^{\circ}$ giving an upper limit on $\theta$ for the particular reflector configuration studied here.

Figure 12 is a plot of the variation in the forces of the edge elements. The force in edge cable 13 initially increases and then decreases. Edge forces 14 and 15 decrease when $\theta$ is increased. This is due to the change in the direction of the tension tie forces.



Figure 11: Variation of forces in net cables

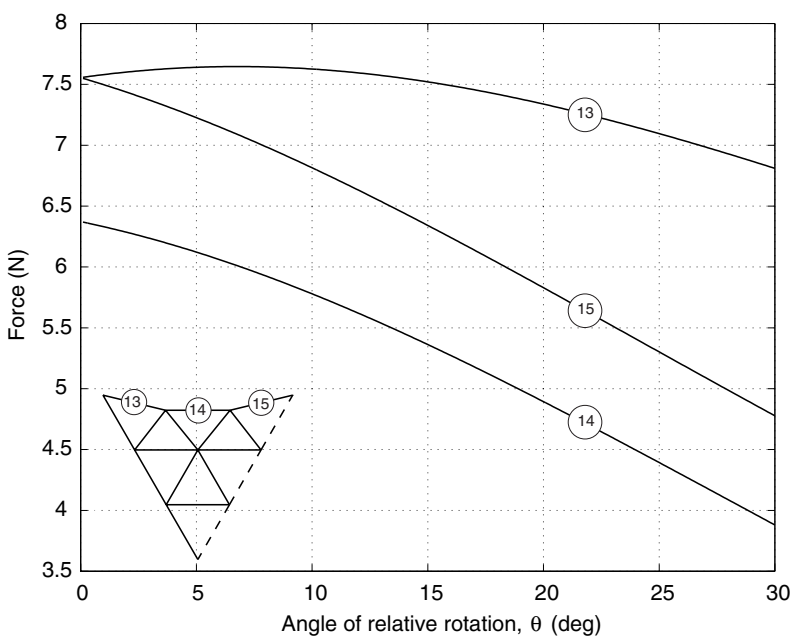

Figure 12: Variation of forces in edge cables.

\section{Ring forces}

The element forces in the ring structure vary exponentially with $\theta$, Figure 13. For small angles, the forces are far too large, especially in the struts. It is not until we reach $\theta=10^{\circ}$ that the forces have decreased to an acceptable level. Further rotation decreases the force, although much more slowly, and for the practical limit of $28^{\circ}$, discussed above, the force in the lateral cables is $3.9 \mathrm{~N}$. Note that the structure is statically and kinematically determinate and, therefore, is not dependent on the prestress level for stiffness. However, the cables must be tensioned to a sufficient level that they are able to take compressive loads without going slack.

Also shown in Figure 13 is the variation of the strut length, which is not as dramatic as the strut forces, although shorter struts are preferable.

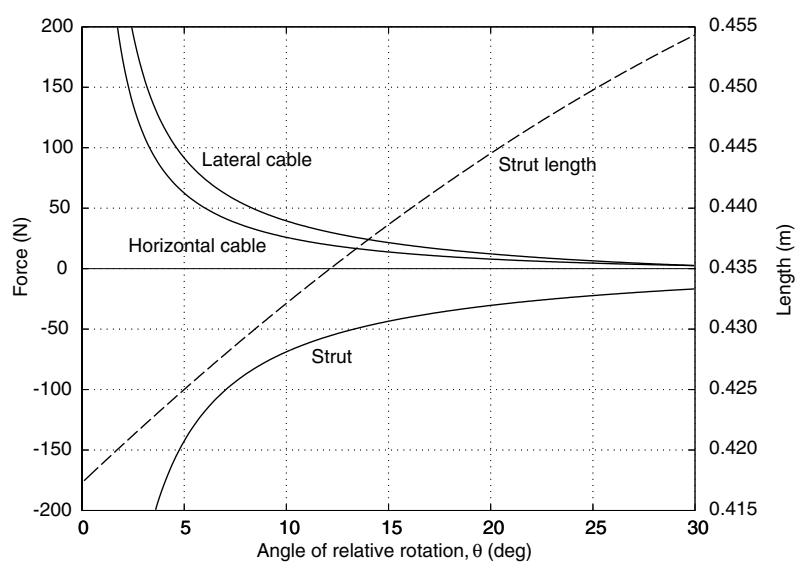

Figure 13: Variation of forces in ring structure.

\section{Additional members}

The additional elements, shown in Figure 2(c), were added to make the structure statically and kinematically determinate but, of course, they need to be pre-tensioned if cables are to be used. In the current configuration of these elements, it turns out that they are always in tension when the hexagons are rotated, Figure 14, and the magnitude of the tension increases almost linearly up to about $10^{\circ}$. However, if the additional members were re-arranged from an anti-clockwise direction (defined from edge joints to ring joints) to clockwise they would be in compression, instead.

\section{Other issues}

Another important issue, not concerned with the force distribution within the structure, is that the struts move closer to the centre of the reflector when $\theta$ is increased. Hence, the struts are more likely to interfere with the tension ties. This might complicate the deployment procedure; therefore, it is important to keep $\theta$ small. 


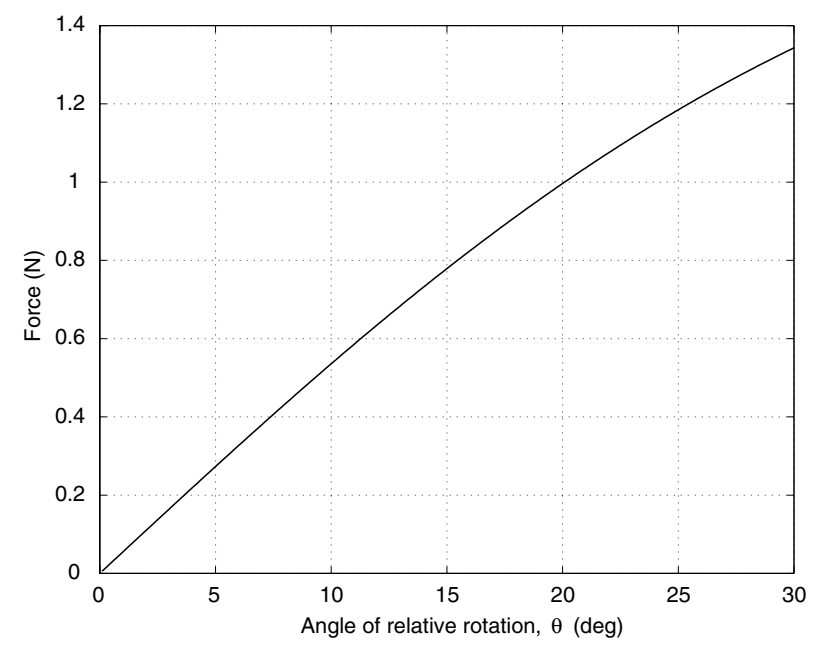

Figure 14: Variation of forces in additional members.

\section{Demonstration Model}

To verify the feasibility of the proposed concept, a smallscale physical model was constructed, with a diameter of $0.47 \mathrm{~m}$.

The nets for the model were constructed on paraboloidal molds of PETG (a thermo-plastic material with the trade name of Vivak) with diameter $D=0.45 \mathrm{~m}$ and focal length $F=0.134 \mathrm{~m}$, on which the position of the nodes of the net had been marked with a 3-axis CNC machine. The elements of the net were $0.8 \mathrm{~mm}$ diameter Kevlar cords which were pretensioned before being taped to the mold; then, the cords were joined at all cross-over points by Nylon loops and bonded with epoxy resin. Corresponding nodes of the two nets were connected with rubber bands, later replaced with steel springs.

Identical Al-alloy, $30 \mathrm{~mm}$ long joint fittings of cylindrical shape with a diameter of $15 \mathrm{~mm}$, were attached to the six corners of each net. These fittings had been precision drilled with $2.0 \mathrm{~mm}$ diameter holes in the directions of all the cords that need to be connected to a node, and all connections were made with epoxy resin. The cords of the ring structure, also attached to the same joint fittings, were made from $1.0 \mathrm{~mm}$ Kevlar cord.

The telescopic struts are $0.46 \mathrm{~m}$ long, each made by cutting off the stick of a foldable umbrella. The umbrella sticks are inserted into $20 \mathrm{~mm}$ long, $6.4 \mathrm{~mm}$ diameter holes that are co-axial with each fitting and fastened with a grub screw. The structure can be easily folded and deployed by hand, photographs are shown in Figures 15-17.

The model works quite well, considering that it was the first attempt at putting together a structure of this kind. However, some of the net cables are slack and there is some interference between the nets and the struts, because the diameter of the net-as manufactured - turned out to be bigger than expected. Correcting these problems should not be difficult when a new model is made.

Figure 17 shows the very compact packaged configuration; note the elongated shape of the package, compatible with the requirements.


Figure 15: Top and bottom views of model structure, expanded. 


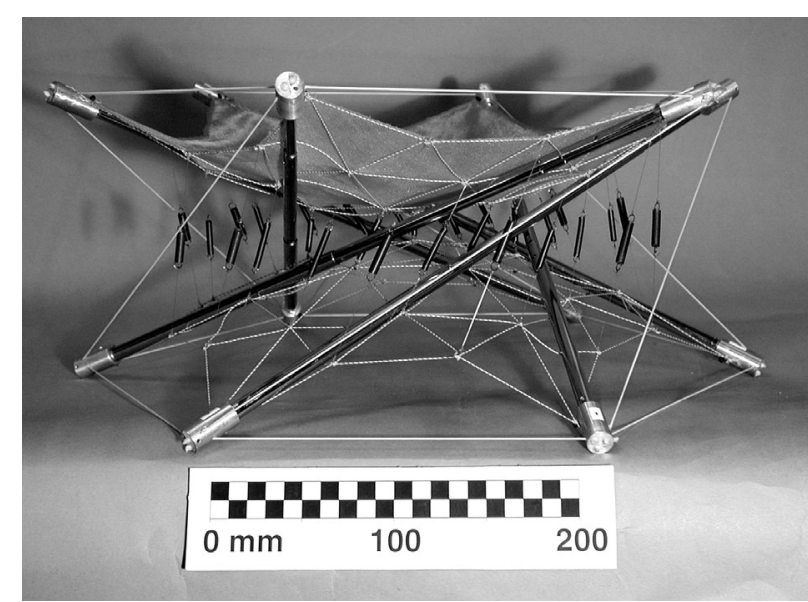

Figure 16: Side view of model structure, expanded.

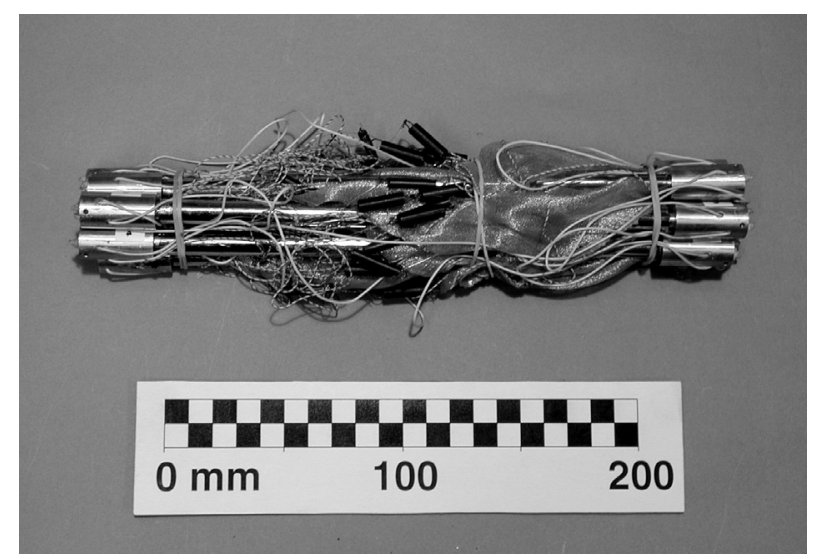

Figure 17: Model structure, folded.

\section{Preliminary Design of $3 \mathrm{~m}$ Reflector}

In this section we determine the main characteristics of a reflector to meet the requirements: $D=3 \mathrm{~m}, F=1.2 \mathrm{~m}$, operation at $10 \mathrm{GHz}$. In particular, we aim to estimate the mass of the reflector.

\section{Network Density}

The surface error of the reflector will originate from a number of different sources, such as thermal distortion of the structure, etc. Only one contribution to the overall error budget can be considered at this stage, namely the effect of approximating the required paraboloid with a polyhedral surface. Therefore, it will be conservatively required that the root-mean-square error $\delta_{\text {rms }}$ should be less than about $1 / 100$ of the wavelength.

At $10 \mathrm{GHz}$ the wavelength is $30 \mathrm{~mm}$ and so the allowable error is $0.3 \mathrm{~mm}$. For a spherical surface of radius $R$, Agrawal et al. (1981) have obtained the following re- lationship between $\delta_{\text {rms }}$ and the side length, $L$, of the triangles

$$
\delta_{\mathrm{rms}}=\frac{L^{2}}{8 \sqrt{15} R}
$$

For a shallow paraboloid the radius of curvature is approximately twice the focal length $F$, thus

$$
R \approx 2 F
$$

For $D=3.0 \mathrm{~m}$ and $F=0.4 D=1.2 \mathrm{~m}$ Equation 9 can be solved for $L$ and yields

$$
L=0.15 \mathrm{~m}
$$

Thus, the number of triangles across a $3 \mathrm{~m}$ diagonal of the hexagon will be 20 , which means that there will be 10 rings of equilateral triangles. The corresponding total length of the cables that make up both nets is $\approx 300 \mathrm{~m}$.

It is assumed that the members of the cable nets are made from graphite composite tapes (density $1740 \mathrm{~kg} / \mathrm{m}^{3}$ ) with a rectangular cross section of $5.0 \mathrm{~mm}$ by $0.2 \mathrm{~mm}$. The weight of the joints in the net is accounted for by doubling the density of the tapes to $3480 \mathrm{~kg} / \mathrm{m}^{3}$. The total mass of the two nets is $1.04 \mathrm{~kg}$.

\section{Mesh}

The reflective mesh is knitted gold-plated Molybdenum wire with a surface density of $0.025 \mathrm{~kg} / \mathrm{m}^{2}$. To account for seams and surface treatment this value is doubled to $0.05 \mathrm{~kg} / \mathrm{m}^{2}$.

Approximating the mesh area with the area of a spherical cap, we have

$$
A=2 \pi R H
$$

where $R$ is the radius of the sphere, hence $R=2 F=$ $2.4 \mathrm{~m}$, and $H$ is the height of the cap; hence $H=0.469 \mathrm{~m}$. Thus, $A=7.07 \mathrm{~m}^{2}$ and the corresponding mass is $0.35 \mathrm{~kg}$.

\section{Force in Springs}

The tension in the mesh applies a lateral loading on the cable net to which it is attached, because the mesh forms a small kink, of angle $L / R$ at the cross-over between adjacent triangles, see Figure 18(a). To prevent the sides of the triangles from becoming significantly distorted, the tension $T$ in the cables of the net must be significantly larger than the transverse load.

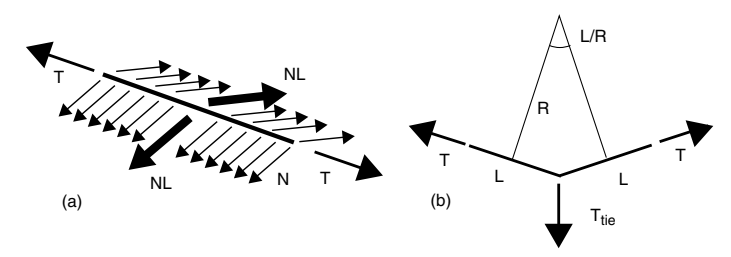

Figure 18: (a) load on cable; (b) equilibrium of a node. 
For a preliminary estimate $T$ will be set equal to ten times the mesh tension $N$ multiplied by the triangle side length $L$. Taking $N=2.0 \mathrm{~N} / \mathrm{m}$ the required tension in the net cables is $T=3.0 \mathrm{~N}$. The force in the tension ties that is required to obtain the specified tension $T$ in the cables is, see Figure 18(b) but note that only two of the six cables connected to this node are shown,

$$
T_{\text {tie }}=3 T L / R=3 \times 3.0 \times 0.150 / 2.4=0.56 \mathrm{~N}
$$

It is interesting to note that the average pressure on the net, given by $T_{\text {tie }}$ over the corresponding area of mesh is $29 \mathrm{~N} / \mathrm{m}^{2}$. This pressure is considerably larger than the self-weight of the mesh under gravity, which is $0.5 \mathrm{~N} / \mathrm{m}^{2}$.

\section{Ring Structure}

The cable net analysed in the section Configuration of Tensegrity Reflector consisted of only three rings of triangles while the current one has ten rings. A preliminary estimate of the loads transmitted to the ring structure by the full-size net can be obtained by assuming that each cable in the three ring reflector represents 3.3 cables in a ten ring reflector. So, we can calculate the forces in the supporting structure by scaling the forces applied by the three ring net, which gives equivalent forces in the tension ties of $8 \mathrm{~N}$. Table 1 lists the forces and length of the members of the ring structure.

\begin{tabular}{lrr}
\hline Element & Force $(\mathrm{N})$ & Length $(\mathrm{m})$ \\
\hline Horizontal cable & 310 & 1.50 \\
Lateral cable & 376 & 1.96 \\
Strut & -712 & 2.88 \\
\hline
\end{tabular}

Table 1: Forces and lengths of elements of ring structure.

\section{Design of Struts}

The struts are designed to resist Euler buckling, subject to a minimum slenderness, $L_{e} / r$, of 200 . Here, $L_{e}$ is the effective length and $r$ the radius of gyration. For a thin-walled tube of radius $R$

$$
r=\sqrt{\frac{I}{A}}=\sqrt{\frac{\pi R^{3} t}{2 \pi R t}}=\frac{R}{\sqrt{2}}
$$

Since $L_{e}=2.88 \mathrm{~m}$, this yields $R>0.0204 \mathrm{~m}$.

Graphite fibre tubes $\left(E=227.5 \mathrm{GN} / \mathrm{m}^{2}\right.$ and $\rho=$ $1740 \mathrm{~kg} / \mathrm{m}^{3}$ ) with an outer diameter of $42 \mathrm{~mm}$ and wall thickness of $0.5 \mathrm{~mm}$ are selected and a check on the buckling load, $3.8 \mathrm{kN}$, is amply satisfied. The total length of these struts is $17.3 \mathrm{~m}$ and the total mass $1.96 \mathrm{~kg}$.

\section{Cable Dimensions}

We assume that graphite fibre is the material used also for the cables of the ring structure. Its tensile strength is $2800 \mathrm{~N} / \mathrm{mm}^{2}$ and a design strength of $500 \mathrm{~N} / \mathrm{mm}^{2}$ is assumed. The maximum cable force is $1880 \mathrm{~N}$, assuming a factor of safety of 5 . Thus, the required cross-sectional area is $3.76 \mathrm{~mm}^{2}$ and, since the total length of the cables in the ring structure is $29.8 \mathrm{~m}$, their mass is $0.19 \mathrm{~kg}$.

\section{Connections and Hinges}

With a length of $2.88 \mathrm{~m}$, each strut must be collapsed to less than a quarter of its length to fit into the launch envelope. One solution would be to use bi-stable composite tubes (Iqbal and Pellegrino 2000) or, alternatively, graphite fibre tubes with three self-locking hinges per strut.

The latter solution is easier to quantify at the present time and so a preliminary mass estimate was obtained by considering the mass of recently developed demonstrator hardware. The tape-spring-rolamite hinges developed by Pellegrino et al. (2000) have a total mass, including attachments to the struts, of $0.2 \mathrm{~kg}$. For the end connections between the strut and the ring structure cables a mass of $0.1 \mathrm{~kg}$ per strut was assumed.

\begin{tabular}{lccc}
\hline Element & Quantity & Unit mass & Mass $(\mathrm{kg})$ \\
\hline Net cables & $300 \mathrm{~m}$ & $0.0087 \mathrm{~kg} / \mathrm{m}$ & 1.04 \\
Struts & $17.3 \mathrm{~m}$ & $0.113 \mathrm{~kg} / \mathrm{m}$ & 1.96 \\
Ring cables & $29.8 \mathrm{~m}$ & $0.0066 \mathrm{~kg} / \mathrm{m}$ & 0.19 \\
Hinges & 18 & $0.20 \mathrm{~kg} / \mathrm{item}$ & 3.60 \\
Connections & 12 & $0.050 \mathrm{~kg} / \mathrm{item}$ & 0.60 \\
Mesh & $7.07 \mathrm{~m}^{2}$ & $0.050 \mathrm{~kg} / \mathrm{m}^{2}$ & 0.35 \\
\hline Total & & & 7.74
\end{tabular}

Table 2: Mass estimates for $3 \mathrm{~m}$ diameter reflector.

\section{$\underline{\text { Discussion and Conclusions }}$}

The proposed reflector concept offers a viable solution to the requirements. Of course, a number of important aspects have yet to be considered, such as the attachment of the reflector to the spacecraft and the deployment sequence of the reflector.

Based on the estimates in Table 2 the total mass of a $3 \mathrm{~m}$ reflector with $F / D=0.4$ is estimated at around $8 \mathrm{~kg}$.

In concluding, it is noted that the proposed concept is - in principle - suitable also for offset configurations, although no detailed study has yet been done.

\footnotetext{
${ }^{\dagger}$ It is assumed that the surface associated with one node is twice the area of a triangle.
} 


\section{Acknowledgments}

The work presented in this report was partially supported by research contract no. CU009-0000004842 between the Defence Evaluation Research Agency and the University of Cambridge, on behalf of the British National Space Centre.

Financial support from The Royal Swedish Academy of Sciences for AGT's visit to the Deployable Structures Laboratory, University of Cambridge, during the year 2000 is gratefully acknowledged.

\section{Appendix: Mesh Generation Procedure}

This appendix describes the procedure used for generating the triangular mesh of the paraboloidal cable nets. The procedure is applicable to nets forming any regular polygon and is illustrated in Figure 19. First, an $m$-sided polygon is divided into $m$ sectors, Figure 19(a). Each sector is then subdivided into $n \times n$ triangles and the edge nodes are projected onto parabolas with the required sag, Figure 19(b). Finally, the triangular mesh is projected onto the required paraboloidal surface giving the shape of the cable net, Figure 19(c).

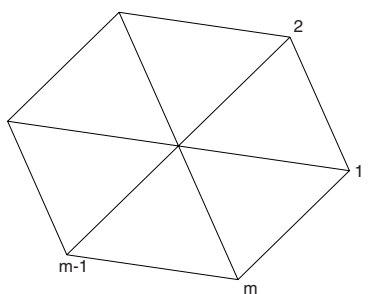

(a)



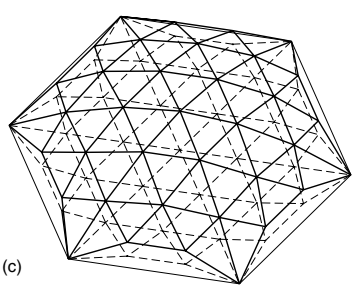

Figure 19: Generation of net; (a) $m$ sided polygon; (b) subdivision of order $n$; (c) vertical mapping onto paraboloid.

In the final net the number of triangles $t$, elements $b$ and joints $j$ are respectively

$$
\begin{aligned}
& t=m n^{2} \\
& b=m \frac{n(1+3 n)}{2} \\
& j=1+m \frac{n(1+n)}{2}
\end{aligned}
$$

The subdivision of order $n$ of a triangular sector is defined by the number of polygon sides, $m$, the radius, $R$, and the two-dimensional sag-to-span ratio, $\rho$, defined as, Figure 20

$$
\rho=\frac{\delta}{2 R_{0} \tan (\theta / 2)}
$$

where $\delta$ is the sag, $\theta=2 \pi / m$, and $R_{0}$ the "effective" radius of the net. Note that the span used in the definition, $2 R_{0} \tan (\theta / 2)$, is different from the distance between the outer vertices which is $2 R \tan (\theta / 2)$.



Figure 20: Triangular subdivision of a sector, here $n=3$.

Given the sag-to-span ratio, $R_{0}$ is calculated by subtracting from $R$ the following lengths, Figure 20

$$
\begin{aligned}
& \Delta_{1}=R \frac{1-\cos (\theta / 2)}{\cos (\theta / 2)} \\
& \Delta_{2}=\frac{\delta}{\cos (\theta / 2)}
\end{aligned}
$$

From Equations 15-17, the relation between $R$ and $R_{0}$ is written as

$$
\frac{R}{R_{0}}=\frac{1+2 \rho \tan (\theta / 2)}{\cos (\theta / 2)}
$$

The radius $R$ is divided into $n$ equal parts, corresponding to $n-1$ rings of identical triangles. In the outer ring, the triangles are distorted by the sag of the edge cables. The edge joints are equidistantly positioned on an arc with radius $r$ and opening angle $\gamma$, Figure 20

$$
\begin{aligned}
& r=\frac{\delta^{2}+R^{2} \sin ^{2}(\theta / 2)}{2 \delta} \\
& \gamma=2 \arccos \frac{r-\delta}{r}
\end{aligned}
$$


The horizontal projection of the length of the edge elements is $2 r \sin (\gamma / 2 n)$. It should also be noted that for odd values of $n$ the actual two-dimensional sag of the edge elements will be slightly less than $\delta$, as shown in Figure 20 for $n=3$.

\section{References}

Agrawal, P. K., Anderson, M. S. and Card, M. F. (1981). Preliminary design of large reflectors with flat facets. IEEE Transactions on Antenna and Propagation, AP-29, 688-694.

Calladine, C. R. (1978). Buckminster Fuller's Tensegrity structures and Clerk Maxwell's rules for the construction of stiff frames. International Journal of Solids and Structures, 14, 161-172.

Duffy, J., Rooney, J., Knight, B. and Crane, C. D. (2000). A Review of a Family of Self-Deploying Tensegrity Structures with Elastic Ties. The Shock and Vibration Digest, 32, 100-106.
Iqbal, K. and Pellegrino, S. (2000). Bi-stable composite shells. In: Proc. 41st AIAA/ASME/ ASCE/AHS/ASC Structures, Structural Dynamics and Materials Conference, 3-6 April 2000, Atlanta GA, AIAA 2000-1385.

Miura, K. (1986). Concept of tension activated cable lattice antenna. In: Proc. 37th IAF Congress, 4-11 October, Innsbruck, Austria, IAF-86-206.

Pellegrino, S. (1993). Structural computations with the Singular Value Decomposition of the equilibrium matrix. Int. J. Solids Structures, 30, 3025-3035.

Pellegrino, S., Kukathasan, S., Tibert, G. and Watt, A. M. (2000). Small satellite deployment mechanisms. Department of Engineering, University of Cambridge, Cambridge Report CUED/D-STRUCT/TR190.

Thomson, M. W. (1997). The AstroMesh deployable reflector. In: Proc. Fifth International Mobile Satellite Conference (IMSC'97), 16-18 June 1997, Pasadena, CA pp 393-398. JPL Publication 97-11. 